\title{
Hydrogen Liquefaction Plant at the Royal Society Mond Laboratory
}

\author{
By Prof. P. Kapitza, F.R.S., and Dr. J. D. CockCRoft
}

$\mathrm{I}^{\mathrm{N}}$ $\mathrm{N}$ the Royal Society Mond Laboratory which is now under construction at Cambridge, in addition to the apparatus required for producing intense magnetic fields, a plant for cryogenic work will be installed. We have developed as a first instalment of this apparatus a hydrogen liquefier which differs from existing liquefiers in that it allows hydrogen of a lower degree of purity than the normal to be used for liquefaction. We propose in this article to give a brief description of the liquefaction apparatus, and later to publish a more complete account.

As is well known, the only method used at present for the liquefaction of hydrogen is essentially the same as that employed originally by Dewar in 1898, and is based on the Joule-Thomson effect combined with a thermal regenerator. In order to obtain a positive Joule-Thomson effect for hydrogen a preliminary cooling with liquid air is required. The principal difficulty encountered in liquefying hydrogen in large quantities is due to the impurities present in the gas; unless extremely pure hydrogen is used, these impurities solidify at the temperature of liquid hydrogen and block the tubes in the regenerator and stop the circulation of the gas. The seriousness of this effect can be shown from a numerical example. The purest commercial hydrogen available in Great Britain is 99.5 per cent pure; 3.9 cubic metres of gas are required to produce 5 litres of liquid hydrogen, and the impurities-chiefly air-when solidified have a volume of about nineteen cubic centimetres. Their deposition is thus quite sufficient to block the small bore tubes of the regenerator, and special precautions have to be taken to avoid this difficulty. In the method developed by Kamerlingh Onnes at Leyden, a preliminary purification of the hydrogen to a high degree of purity is carried out, and steps are taken to save the gas after it has been used for experiments. Another method introduced by Meissner is to make a special trap in the hydrogen circulation in such a way that in it the greater part of the impurities are condensed ; the liquefaction is stopped at intervals to allow the trap to be warmed by a special electrical heater and emptied.

In our liquefier we have adopted a different principle which allows us to use the apparatus continuously even with commercial hydrogen. The method is as follows: Two hydrogen circuits are used in the liquefaction process ; one circulation is similar to that used in ordinary liquefiers, but is completely closed; in it about 0.7 cubic metre of purified hydrogen is compressed to $160-170$ atmospheres, and after preliminary cooling in liquid nitrogen at reduced pressure passes through a regenerator spiral and then expands to normal pressure, thereby cooling down a 'condenser' to liquid hydrogen temperature, after which it returns through the regenerator to the compressor. In the second circuit we use ordinary commercial hydrogen, which is reduced to a pressure of $3-4$ atmospheres by means of a reduction valve from a cylinder. This hydrogen is cooled down to the temperature of liquid nitrogen and is then passed directly into an 'exchanger' cooled by the first circuit. Since the liquefaction temperature of hydrogen at 3 atmospheres pressure is a few degrees higher than at normal pressure, it liquefies in the exchanger. The whole of the cooling down process, from the temperature of liquid nitrogen to the temperature of liquid hydrogen, takes place in the exchanger, so that all the impurities condense here and do not have a chance of being deposited in the tubes. The solidified impurities are heavier than the liquid hydrogen and remain at the bottom of the exchanger, which is large enough to retain all the impurities solidified in the course of a run.

The scheme of the liquefier is shown diagrammatically in Fig. 1, and a photograph of the liquefier in Fig. 2. The hydrogen in the closed circulation enters at tube 1 , passes through the regenerator spiral $A$, and enters the container $B$ containing liquid nitrogen at reduced pressure, from which it passes through the second regenerator spiral $D$, and then through the expansion valve $E$ to the condenser $F$, where it is partially liquefied. When the condenser $F$ is about one-third full, the liquid hydrogen passes through tube 6 to a spiral which cools exchanger $G$; it then

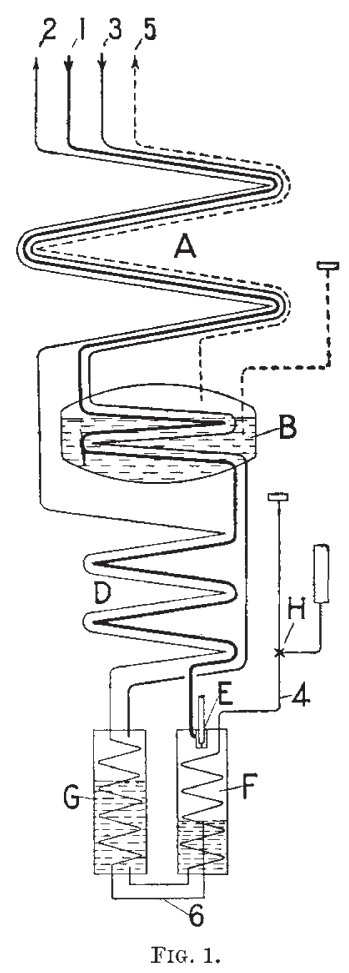
goes through the regenerator spirals $D$ and $A$ and passes back to the compressor through the outlet 2. The commercial hydrogen enters the tube 3 and passes only through the first regenerator circuit $A$, and the container $B$, and then passes directly into the exchanger $G$, where it meets the spiral cooled by the closed circulation and is liquefied at three atmospheres pressure. The exchanger $G$ is continuously drained through the tube 4 passing through the condenser $F$ as a spiral and leading to valve $H$, which controls the flow of liquid hydrogen into a Dewar receptacle. In passing through the spiral of condenser $F$, the liquid hydrogen is cooled through a few degrees from the temperature corresponding to liquefaction at three atmospheres pressure to the temperature of liquid hydrogen at atmospheric pressure. This prevents excessive evaporation when it is 
drawn off into the Dewar receptacle. The exchanger $G$ is filled with wire gauze which helps in the liquefaction. In the actual liquefier a double container is used for the liquid nitrogen. In one part the liquid nitrogen is evaporated at slightly reduced pressure and the evaporated gas passes up tube 5 of regenerator $A$ and takes up sufficient heat to cool down the incoming hydrogen of the liquefaction circulation; in the other part the liquid nitrogen evaporates at a few centimetres pressure and passes away directly to the pump. (During a recent visit to Dr. Simon's laboratory at Berlin, we have been informed that the same principle of

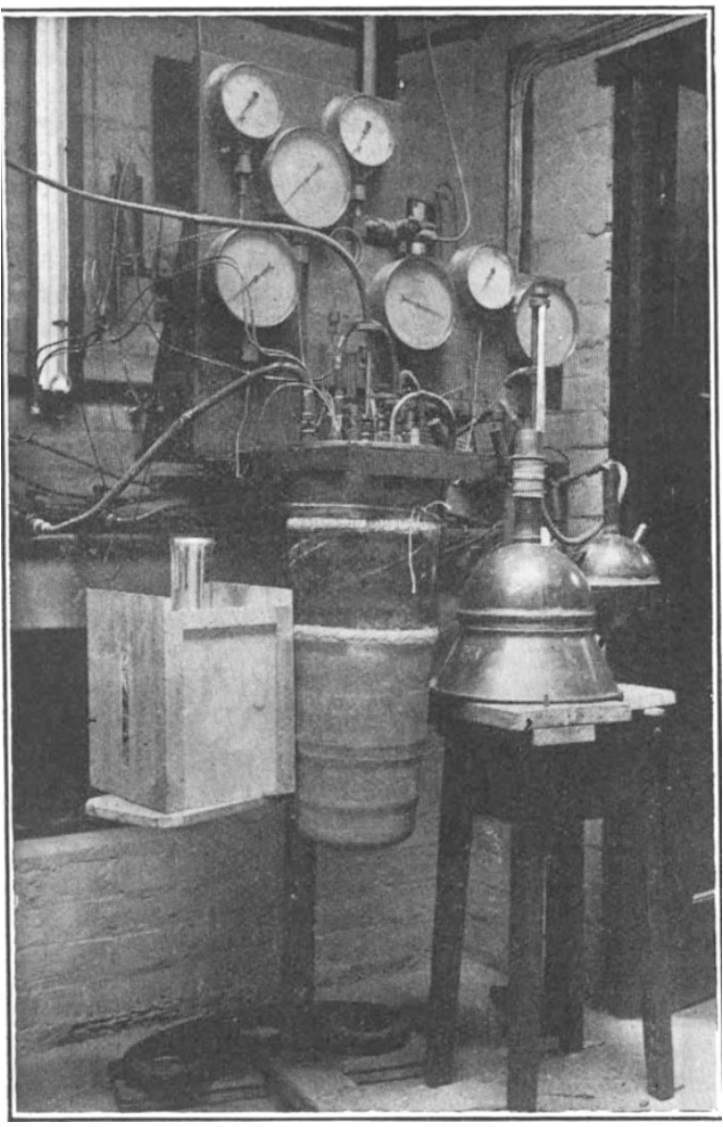

FIG. 2.

cooling hydrogen in two stages was used in his liquefier.)

The tubes used in the liquefier have a small crosssection and the gas is allowed to flow with high velocity. We find, as suggested by Meissner, ${ }^{1}$ that the heat exchange can be calculated from Nusselt's formula. ${ }^{2}$ From this formula it appeared that a good heat exchange could be obtained by using a high velocity of the gas, and this enabled us to reduce the length of the regenerator spirals very considerably. In our case the spiral $A$ was about 2.5 metres long and the spiral $D 5$ metres. The high tensile strength of the copper-nickel alloy allows the heat capacity of the regenerator spiral to be considerably reduced. The whole apparatus is soldered into a copper vessel $26 \mathrm{~cm}$. in diameter by $67 \mathrm{~cm}$. high, which is then evacuated. By the use of charcoal to absorb gases given off from the metal, a good vacuum can be maintained and a high degree of thermal insulation maintained without the use of glass Dewar vessels.

The new liquefier has the considerable advantage that the rate of liquefaction can be measured continuously by a flow meter placed before the inlet of tube 3. The flow meter will also indicate the moment when the gas begins to condense. An electrical flow meter is used. The hydrogen is passed through a thin-walled copper-nickel tube $20 \mathrm{~cm}$. long, which is heated in the middle by a platinum wire spiral. The cooling of this spiral depends on the rate of flow of hydrogen through the tube and is measured by its change of resistance. The spiral is made in two parts which form the two opposite arms of a Wheatstone bridge; the other two arms are also made of platinum, and are wound on the thick copper main tube, and thus are kept at the temperature of the inlet hydrogen. The apparatus is calibrated by separate experiments.

The liquefier has proved to be as efficient as ordinary existing liquefiers. The heat exchange is good, and the temperature of the return hydrogen and nitrogen at exit is only $10^{\circ}$ below the temperature of the inlet hydrogen. 4-5 litres of liquid nitrogen are used for the initial cooling, and liquefaction starts $40-50$ minutes after the beginning of the preliminary cooling. The liquefaction rate is 4 litres an hour, of which about twenty per cent is lost when the hydrogen is drawn off. The liquid nitrogen consumption is about $1 \cdot 3$ litres per net litre of liquid hydrogen. We normally produce 6 litres of liquid hydrogen in a single run, using commercial hydrogen, and have not up to the present had a single stoppage due to blocked tubes. The liquefier was made in our workshop by the laboratory mechanic, Mr. H. E. Pearson.

The compressor used for the work is a compact, triple stage, high speed (600 r.p.m.) machine which was specially built for us by Messrs. Reavell and Company. The detailed design was worked out by Mr. J. Hendry. Special care was taken to ensure complete freedom from leakage; the space below the cylinders is connected to the compressor suction, and the piston rods are surrounded by oilsealed glands which are fitted with leakage indicators. At full speed the compressor capacity is 25 cubic metres of free gas per hour; at present we are using it at reduced speeds giving a delivery of 16 cubic metres per hour.

The problem of storage of liquid hydrogen has been dealt with on new lines. It is well known that owing to the small latent heat it is difficult to store liquid hydrogen in an ordinary Dewar flask for more than a day. As described by Meissner, the best containers, of a capacity of 6 litres, evaporate at the rate of $15 \mathrm{gm}$. (200 c.c.) of hydrogen an hour ; these containers have a specially good vacuum and silvered surfaces to prevent radiation losses, which are the most important source of loss of cold. In order to diminish these radiation losses further, we have worked out a design for a Dewar flask which has all the advantages of the

No. 3250, VoL. 129] 
method of keeping the liquid hydrogen container immersed in liquid air, without the disadvantages and technical difficulties which occur if this method is used on a large scale. A drawing of the flask is shown in Fig. 3. It consists of a twin flask ; flask

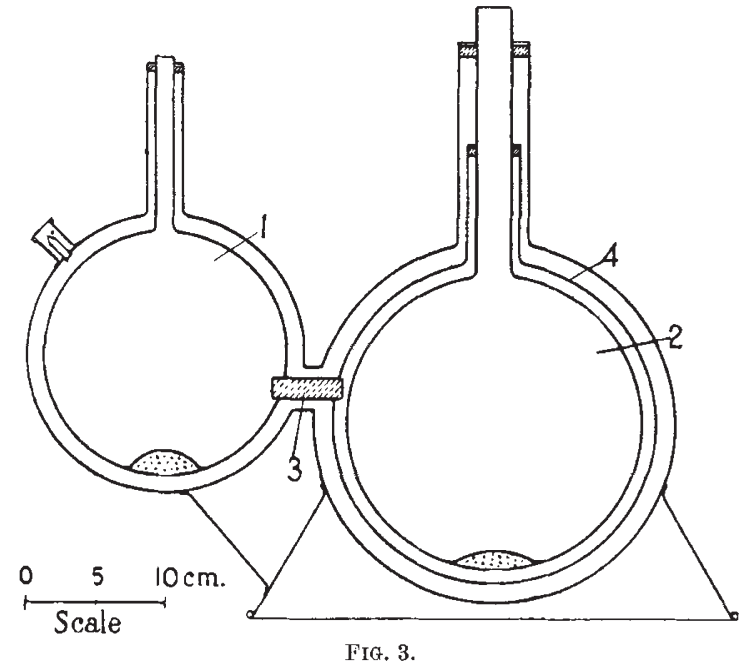

1 contains liquid air, and flask 2 liquid hydrogen. The inside of flask 1 is connected by means of a copper rod 3 to a copper shield 4 which surrounds the container of flask 2. The liquid air cools the shield 4 by thermal conductivity, and the radiation losses from the liquid hydrogen are considerably reduced. The hydrogen flask has a capacity of 5 litres and the liquid air flask holds 2 litres. The evaporation of hydrogen is about $2.5 \mathrm{gm}$. an hour, which is about seven times smaller than for ordinary flasks, and enables the liquid hydrogen not required for immediate use to remain in the flask for five days. The consumption of liquid air is about 1.7 litres per day. A picture of the flask will be seen in Fig. 2 standing beside the liquefier.

Another flask is now being constructed in which the radiation will be further reduced by silvering the inner surfaces, thereby increasing the efficiency of the flask still further. The flask was manufactured for us by Messrs. Siebe Gorman and Co.,Ltd.

The remaining equipment of the Laboratory is on the usual lines, and all the known precautions against explosion are employed, flame-proof mining type motors being used for the compressor drive and all open switchgear placed in a separate room. Besides the usual precautions we have introduced one more, which consists of a standard alarm lamp as used for showing the presence of coal gas in mines. This indicator is manufactured by Messrs. 'The W.R. Patents, Ltd. Should a leak occur, and the concentration of hydrogen in the room reach a value of more than one per cent, the lamp operates a relay which automatically stops the machines, and throws the windows open at the bottom and the top of the rooms where the liquefier is in operation, thus providing a complete air circulation.

We were enabled to construct this plant by a special grant made by the Department of Scientific and Industrial Research.

\footnotetext{
1 " Handbuch der Physik", vol. 11, p. 295

3 Z. Instrumentenkunde, 50,121; 1930 .
}

\section{Diagnosis of Smallpox*}

$17 \mathrm{OR}$ adequate control of infectious disease, early and accurate diagnosis is of great importance, but clinical differentiation is not always easy in the early stages. Thus smallpox, even of the severe type, may be mistaken for chickenpox. Some years ago M. H. Gordon described a "flocculation' test for smallpox, which was later investigated by W. L. Burgess, J. Craigie, and W. J. Tulloch : the last two authors have now extended and amplified the earlier observations. The technique of the test is, in brief, as follows: crusts obtained from the pocks are dried and finely powdered; the powder is extracted with ether and then with saline for some hours; the mixture is frozen, thawed, and centrifuged to remove particulate matter. Series of dilutions of the extract are made in saline : to one antivaccinia serum is added, to the other normal rabbit serum, and both sets are incubated for about 18 hours at $45^{\circ}-55^{\circ} \mathrm{C}$. Flocculation occurs in the first series at high dilutions of the extract if the crusts were obtained from a case of smallpox, but is not seen in the second series to which normal rabbit serum was added.

The authors carried out an investigation of every step in the test so as to obtain the best conditions:

* Medical Research Council. Special Report Series No. 156 Further Investigations on the Variola-Vaccinia Flocculation Reaction. By J. Craigie and W. J. Tulloch. Pp. 129. (London: H.M. Stationery Oflice, 1931.) $3 s$. net. thus it was found that more satisfactory results were obtained when the concentration of antiserum was kept constant and that of the antigen (crust extract) varied than when the converse system was employed. For the extraction of the crusts, 0.9 per cent sodium chloride solution was better than weaker concentrations and much more satisfactory than a phosphate buffer solution : in the latter the reaction shifted towards the acid side, whereas that of the saline extracts is remarkably stable. It is essential to carry out the flocculation test at about $p \mathrm{H} 7$ : at $p \mathrm{H} 6$ or 8 non-specific sedimentation is liable to occur. The preliminary extraction with ether removes certain substances which may obscure the test and also reduces the tendency to anomalous flocculation. It has been found further that the flocculable substance is fairly intimately connected with the euglobulin fraction of extracts although it can be to some extent separated therefrom: when the flocculable substance has been destroyed infectivity has also disappeared, but the latter can be markedly reduced without affecting the former. The purified euglobulin fraction is not very stable to heat but is fairly stable to low concentrations of phenol. It is possible to detect by the flocculation test so little as $0.0000005 \mathrm{gm}$. of purified flocculable material.

The flocculation test is positive in smallpox

No. 3250 , VoL. 129] 